\title{
Acute effects of high-intensity interval, resistance or combined exercise protocols on testosterone - cortisol responses in inactive overweight individuals
}

\author{
By \\ FT. Gina P. Velasco-Orjuela \\ A thesis submitted to the School of Medicine and Health \\ Science of Universidad del Rosario \\ in partial fulfillment of the requirements for the \\ degree of Master of Physical Activity and Health \\ Robinson Ramírez-Vélez (Advisor) \\ Jorge Enrique Correa-Bautista (Co-advisor) \\ Center of Studies in Physical Activity Measurements, \\ School of Medicine and Health Sciences, \\ Universidad del Rosario, Bogotá D.C, Colombia \\ Mikel Izquierdo (International co-advisor) \\ Department of Health Sciences, Public University of Navarre, CIBER de Fragilidad y \\ Envejecimiento Saludable (CB16/10/00315), Tudela, Navarre, Spain.
}

Anthony C. Hackney (International co-advisor)

Endocrine Section-Applied Physiology Laboratory, University of North Carolina at Chapel Hill, Chapel Hill, USA.

Eduardo L. Cadore (International co-advisor)

Exercise Research Laboratory, Physical Education School, Federal University of Rio

Grande do Sul, Porto Alegre, RS, Brazil.

Center of Studies in Physical Activity Measurements

School of Medicine and Health Sciences

Universidad del Rosario

Bogotá D.C,

Colombia 2018 


\section{Distribution Agreement}

In presenting this thesis or dissertation as a partial fulfillment of the requirements for an advanced degree from Universidad del Rosario, I hereby grant to Universidad del Rosario and its agents the non-exclusive license to archive, make accessible, and display my thesis or dissertation in whole or in part in all forms of media, now or hereafter known, including display on the world wide web. I understand that I may select some access restrictions as part of the online submission of this thesis or dissertation. I retain all ownership rights to the copyright of the thesis or dissertation. I also retain the right to use in future works (such as articles or books) all or part of this thesis or dissertation. 


\section{ABSTRACT}

PURPOSE: The purpose of this study was to compare the hormonal responses to one session of high-intensity interval training (HIIT, $4 \times 4$ min intervals at $85-95 \%$ maximum heart rate [HRmax], interspersed with 4 min of recovery at 75-85\% HRmax), resistance training (RT at 50-70\% of one repetition maximum 12-15 repetitions per set with 60s of recovery) or both (HIIT+RT) exercise protocol in a cohort of physical inactivity, overweight adults (age 18-30 years old).

METHODS: Randomized, parallel-group clinical trial among fifty-one men $(23.6 \pm 3.5 \mathrm{yr}$; $83.5 \pm 7.8 \mathrm{~kg} ; 28.0 \pm 1.9 \mathrm{~kg} / \mathrm{m} 2$ ), physical inactivity (i.e. <150 min of moderate-intensity exercise per week for greater than 6 months), with abdominal obesity (waist circumference $\geq 90 \mathrm{~cm}$ ) or body mass index $\geq 25$ and $\leq 30 \mathrm{~kg} / \mathrm{m} 2$ were randomized to the following 4 groups: high-intensity interval training $(n=14)$, resistance training $(n=12)$, combined highintensity interval and resistance training $(n=13)$, or non-exercising control $(n=12)$. Cortisol, total- and free-testosterone and total-testosterone/cortisol-ratio (T/C) assessments (all in serum) were determined before (pre) and 1-min post-exercise for each protocol session.

RESULTS: Decreases in cortisol levels were -57.08 (95\%CI, $-75,58$ to $-38,58 ; \mathrm{P}=0.001$; $\left.\eta^{2}=0.61\right)$ and $-37.65\left(95 \% C I,-54,36\right.$ to $\left.-20,93 ; \mathrm{P}=0.001 ; \eta^{2}=0.51\right)$ in the HIIT and control group, respectively. Increases in $\mathrm{T} / \mathrm{C}$ ratio were 0.022 (95\% CI, 0.012 to $0.031 ; \mathrm{P}=0.001$; $\left.\mathrm{\eta}^{2}=0.49\right)$ and $0.015\left(95 \% \mathrm{CI}, 0.004\right.$ to $\left.0.025 ; \mathrm{P}=0.007 ; \mathrm{\eta}^{2}=0.29\right)$ in the HIIT and control group, respectively. In per-protocol analyses revealed significant change in cortisol levels [interaction effect $\mathrm{F}(7.777), \eta^{2}=0.33$ ] and $\mathrm{T} / \mathrm{C}$ ratio [interaction effect $\mathrm{F}(5.298), \eta^{2}=0.25$ ] between groups over time.

CONCLUSIONS: The present data indicate a HIIT reduced cortisol and increased totaltestosterone/cortisol-ratio levels significantly in physical inactive adults. Further study is required to determine the biological importance of these changes in hormonal responses in overweight men. 


\section{INTRODUCTION}

Current lifestyle trends characterized by inactivity and poor dietary habits have led to a dramatic decline in the health of the Latin American population (1). Decreased physical activity and sedentary behavior - combined with poor dietary habits - have been implicated as potential contributing factors in the obesity crisis (2). Overweight and obesity may affect metabolic health with hormonal and sex hormone levels abnormalities (3). In obese men before puberty and at its onset, there has been an increase in free testosterone, in response to the decrease in SHBG that occurs in this condition in order to maintain total testosterone levels (4). Two integral endocrine gland hormones greatly affected by obesity are testosterone and cortisol $(3,5)$. These hormones have significant effects on both protein metabolism and lipolysis, two metabolic pathways affected by obesity, but also essential in the management and treatment of obesity and cardiovascular comorbidities $(5,6)$.

Interestingly, physical exercise, particularly physical training is an effective strategy to combat metabolic disorders due to its ability to influence body composition and some biomarkers, such as cholesterol, inflammatory cytokines and insulin resistance $(7,8)$. Highintensity exercise ( $>80 \%$ of peak oxygen uptake), has been shown to produce significant increases in circulating hormones $(9,10)$. Along this line, testosterone and cortisol have been suggested to be important mediators of the exercise-induced hormonal responses, and are considered as useful biomarkers of anabolic and catabolic hormonal control, respectively $(11,12)$. Furthermore, the testosterone/cortisol ratio (T/C ratio) has been suggested to be an indicator of the anabolic/catabolic status (9). Thereby, either an increase in testosterone, a decrease in cortisol, or a combination of both would indicate the potential overall state of anabolism (13).

However, the relationship between the time course of training adaptations during a training cycle and the parallel time-course of potential changes in resting testosterone, cortisol or T/C ratio is not well established. For example, a 14-day mesocycle with frequent highintensity interval 5 training (HIIT) sessions induced both endurance adaptions and increases in serum testosterone concentration in thirteen male junior triathletes (15.8 $\pm 1.8 \mathrm{yr})$ (14). 
West et al. (14) showed an acute increase in concentrations of testosterone and cortisol concentrations among twelve healthy young men $(21.8 \pm 1.2 \mathrm{yr})$, after several resistance exercise sessions. In contrast, others have reported significant adaptive responses in professional cyclists to a training program that also induced declining testosterone and increasing cortisol concentrations indicative of an increased catabolic state (15).

Discrepancies among studies may be due to differences in the baseline training status of participants, or the training dose administered. Furthermore, cortisol increases, while testosterone levels decrease with obesity and several studies have reported the T/C ratio are significantly correlated with age, body mass index (BMI) and waist circumference $(16,17)$. There is considerable information given concerning the endocrinological response in lean individual as a point of reference for future research using an obese model, but exercise endocrine work in obese is warranted due to exercise hormonal response anomalies (18).

Thus, to determine the main factors related to mode of exercise training sessions (i.e., highintensity interval training - HIIT, resistance training - RT, or combined training - HIIT + RT) that greatly influence acute testosterone and cortisol responses, it is important to create an anabolic hormonal response and optimize the adaptations to training. Although strength and aerobic training intensity volume have a critical influence on the magnitude and/or duration of the acute response of testosterone and cortisol (12), to the best of our knowledge, there are no data available regarding the comparison between HIIT, RT or HIIT $+\mathrm{RT}$ on the acute responses of testosterone and cortisol, especially in overweight individuals, who may have risk to altered hormonal responses.

Therefore, the aim of the present study was to compare the acute hormonal responses of cortisol, total- and free-testosterone and total-testosterone/cortisol-ratio at the beginning and after one session of HIIT, PRT or both exercise protocol in a cohort of physical inactivity, overweight adults (age 18-30 years old). It is hypothesized that the HIIT + RT protocol (due to the highest intensity) induces the highest metabolic perturbations and therefore the highest hormonal responses compared to the RT and the HIIT protocol. 


\section{METHODS}

\section{Study design and setting}

The present study is a randomized controlled trial (ClinicalTrials.gov ID: NCT02915913; "BrainFit Study"). The BrainFit Study is a single blind, randomized controlled $2 \times 2$ factorial trial. The study received ethical approval from the Medical Research Ethics Committee of The Universidad Nacional de Colombia (Code $\mathrm{N}^{\circ}$ 018-223-16). Random allocation to treatment is performed at the individual level.

\section{Participants}

Males aged 18-30, physical inactivity (i.e. <150 min of moderate-intensity exercise per week for greater than 6 months, according to an International Physical Activity Questionnaire), with abdominal obesity: waist circumference $\geq 90 \mathrm{~cm}$ or excess weight, body mass index $\geq 25$ and $\leq 30 \mathrm{~kg} / \mathrm{m} 2$, identified as being willing and with almost immediate availability was enrolled. Eligible subjects for the present study and those interested in participating were invited to a pre-test that includes an interview and further assessments, performed at the Centre of Studies in Physical Activity Measurements (in Spanish, CEMA), School of Medicine and Health Sciences, University of Rosario, Bogotá, Colombia. Risks was minimized by ruling out contraindications to the testing and training protocols via a health history and a thorough physical examination prior to the testing sessions. Inclusion and exclusion criteria are provided in Table 1.

Table 1. Inclusion/exclusion criteria

Inclusion criteria

Males aged 18-30

Central obesity: waist circumference $\geq 90 \mathrm{~cm}$ or excess weight: body mass index $\geq 25$ and $\leq 30$ $\mathrm{kg} / \mathrm{m}^{2}$

Interested in improving cardiovascular health and physical fitness

Written informed consent
Systemic infections

Weight loss or gain of $>10 \%$ of body weight in the past 6 months for any reason

Currently taking medication that suppresses or stimulates appetite

Systolic blood pressure $140 \mathrm{mmHg}$ or diastolic blood pressure $90 \mathrm{mmHg}$ 
Gastrointestinal disease, including self-reported chronic hepatitis or cirrhosis, any episode of alcoholic hepatitis or alcoholic pancreatitis within past year, inflammatory bowel disease requiring treatment in the past year, recent or abdominal surgery (e.g., gastrostomy)

Asthma

Diagnosed diabetes mellitus (type 1 or 2 ), fasting impaired glucose tolerance (blood glucose $126 \mathrm{mg} / \mathrm{dL}$ ), or use of any anti-diabetic medications

Currently taking antidepressant, steroid, or thyroid medication, unless dosage instable (no change for 6 months)

Current exerciser (>150 min organized exercise per week)

Indication of unsuitability of current health for exercise protocol (Physical Activity Readiness. Questionnaire, PARQ)

Any other conditions which, in opinion of the investigators, would adversely affect the conduct of the trial

\section{Recruitment}

Consecutive males with abdominal obesity or excess weight were recruited from different educational institutions (Universidad Nacional, Universidad del Rosario, Universidad Santo Tomás, Universidad Manuela Beltrán and Universidad de la Sabana) that received referrals from both medical consultants and biomedical practitioners in the capital district of Bogotá, Cundinamarca Department in the Andean region. Subjects who were interested in participating were approached with further information and (i) initially screened for preparticipation exercise using a cardiovascular and musculoskeletal checklist (i.e., the patient's medical history, disease history, physical fitness and more); (ii) baseline testing; (iii) a single isocaloric acute training protocol and (iv) post-training testing. Participants provided written informed consent prior to enrollment to the larger study.

\section{Blinding and randomization methods}


Randomization into the four study arms was performed by CEMA at the University of Rosario, Bogotá, Colombia, using block randomization with a block size of four. Eligible participants were randomly assigned after completing the baseline measurements to either the control or exercise training groups. The principal investigator coordinated the allocation sequence, and randomization was computer generated. Investigators and statisticians were blinded to treatment allocation throughout the trial protocol. Access to the allocation code was restricted to one study statistician who did not perform the final study analyses. Randomization was conducted independently using sealed opaque envelopes. Moreover, the importance of maintaining the blinding and allocation concealment were reinforced by regularly scheduled conference calls at the sites and daily meetings with the field investigators.

\section{Intervention}

Each of the volunteers participated in four randomized trials (HIIT, RT, HIIT + RT and control [no exercise]), and the starting trial was randomized.

1. Non-exercise group (control): This group followed the usual routines of standard care but were not taught to perform a specific regimen for supervised training and walking exercises; a record of any exercises done that are not a part of this study was kept.

2. High-intensity interval training (HIIT) group: The HIIT protocol was completed with fast walking or running on a treadmill with the deck inclined (i.e. grade) to reach the desired intensity. All HIIT sessions were preceded with a 5-min warm-up and ended with a 4-min cool down at $65 \%$ heart rate reserve until the expenditure of 400 to $500 \mathrm{kcal}$. The HIIT protocol consisted of four bouts of 4-min intervals at 85-95\% HR reserve, interspersed by 4 min of active recovery at $75-85 \%$ HR reserve. Participants in the HIIT groups were instructed to reach the target HR for each interval within the first 2 min of the 4-min interval. We calculate the training energy expenditure for participants' age ranges associated with meeting the consensus public health recommendations from the World Health Organization (19) and the US Department of Health and Human Services (20). During the supervised intervention, we record heart rate (HR) using an HR monitor (Polar Pacer, USA) to ensure compliance with the exercise stimulus at the predetermined target HR zone. In addition, the rating of perceived exertion (RPE, Borg scale) was also be measured in each exercise session (15-17 in high intensity and 11-13 to recovery). 
3. Resistance training (RT) group: The protocol training phase at $50-70 \%$ of one repetition maximum, $1 \times 12-15$ repetitions, $60 \mathrm{~s}$ recovery as many times as needed according to subject weight until the expenditure of 400 to $500 \mathrm{kcal}$. The five upper body exercises included the bicep screw curl, triceps extension, dumbbell side lateral raise, dumbbell front raise and military press. Three lower body exercises included dumbbell squat, dumbbell front lunge, and dumbbell side lunge. HR and Borg RPE were monitored in each exercise session. Each session was preceded and followed by a gradual warm-up and cool-down period (both of 10-min duration and consisting of walking and light, static stretching (avoiding muscle pain) in most muscle groups). The cool- 10 down period also includes relaxation and stretching exercises. Each participant's workloads were prescribed on an individual basis using their one repetition maximum results during the initial orientation. Muscular strength was assessed two days before acute intervention using the one repetition maximum (1RM) test, implemented according to similar procedures (21).The 1RM was performed in five upper body exercises included the bicep screw curl, triceps extension, dumbbell side lateral raise, dumbbell front raise, military press and three lower body exercises included dumbbell squat, dumbbell front lunge, and dumbbell side lunge, carried out in the morning between 9 and $11 \mathrm{~h}$, and the highest load of three attempts were reported per exercise.

4. Combined training (HIIT+RT) group: This group were received both the HIIT and RT protocols as described above. Therefore, the energy expenditure associated with the physical training prescribed for the vigorous intensity group was $\approx 400$ to $500 \mathrm{kcal} / \mathrm{session}$.

\section{Training intensity and energy expenditure during training}

In terms of training intensity, the actual values of intensity were the mean of HR measured in HIIT or combined groups, and the average value of workload and repetitions determined in acute session in RT group, respectively. The exercise training was supervised throughout. Attendance at supervised sessions as well as checks for compliance with target HR and energy expenditure were monitored and recorded by research staff. Energy expenditure was estimated during exercise via indirect calorimetry using a Cosmed K5 b2 portable metabolic system (Rome, Italy) assuming a non-protein respiratory exchange ratio (22). 


\section{Blood draws and analysis}

Participants arrived at the CEMA laboratory between 6:00 and 9:00 AM, following an overnight fast. Participants were reminded to maintain standardized conditions prior to each assessment point which included arriving in a hydrated state having abstained from caffeine and alcohol consumption for $36 \mathrm{~h}$. Serum was then extracted, centrifuged at 3,300 revolutions per minute, aliquoted, and stored at $-80^{\circ} \mathrm{C}$ until subsequent analysis. Concentrations of serum total-free testosterone and cortisol were analyzed by using commercially available electrochemiluminescence immunoassay kits (Roche Diagnostics $\mathrm{GmbH}$, Mannheim, Germany). All analyses were tested in the same assay with standard procedures (Deyi Biomedical Technology Co., Ltd., Beijing, China). Within assay, coefficient of variation of standards and samples were: $7 \%$ and $6 \%$, and $4 \%$, for total testosterone, free testosterone, and cortisol, respectively.

\section{Body composition and performance measures}

After completing another general health status information questionnaire, participants were instructed to wear shorts and a t-shirt to the physical exam. They were also required to remove all worn jewelry and metal objects. Once the subjects were barefoot and in their underwear, their body weight $(\mathrm{kg})$ was measured using an electric scale (Model Seca® mBCA 514 Medical Body Composition Analyzer, Hamburg, Germany) with a range of 0 to $200 \mathrm{~kg}$ and with an accuracy of within $100 \mathrm{~g}$. Height was measured with a portable stadiometer with a precision of $0.1 \mathrm{~mm}$ and a range of $0-2.50 \mathrm{~m}$ (Seca® 274, Hamburg, Germany). BMI was calculated by using the formula proposed by Quetelet where BMI = body mass (kg)/height (m2). Body mass index status was evaluated according to the World Health Organization criteria (23).

The waist circumference (WC) $(\mathrm{cm})$ was measured as the narrowest point between the lower costal border and the iliac crest. When this point was not evident, it was measured at the midpoint between the last rib and the iliac crest, using a metal tape measure (Lufkin W606PM®, Parsippany, New Jersey, USA), in accordance with the International Society for the Advancement of Kinanthropometry guidelines (24). The evaluation process was carried out by a team of professionals (2 physical therapy professors) with extensive experience in anthropometric measurement. The technical error of measurement (TEM) values were less than $2 \%$ for all anthropometric variables. 
Body fat mass was determined for a multifrequency bioelectrical impedance analyser (BIA by a tetrapolar whole body impedance (Model Seca ${ }^{\circledR}$ mBCA 514 Medical Body Composition Analyzer, Hamburg, Germany). A detailed description of the BIA technique can be found in a previous study (25). For the calculation of intra-inter observer TEM, at least 50 subjects needed to be measured (30 men, 20 women, aged 22.3 $\pm 2.1 \mathrm{yr}$ ). The corresponding intra-observer technical error (\% reliability) of the measurements was $95 \%$.

\section{Statistical analysis}

Prior to the planned statistical analyses, preliminary analysis was conducted (Shapiro-Wilk test) to confirm data distribution normality. A two-way mixed analysis of covariance (ANCOVA) with repeated measures was used to test the main effect (i.e., group effect) and the interaction effect (time and group interaction) with the baseline value as covariate on the outcome variables (hormonal responses). Cohen's d for effect size were also calculated to determine the magnitude of the group differences. Effect size were classified as small, medium, and medium-to-large effects $(<0.20,0.2-0.6$ and $0.6-1.2$, respectively), and partial $\eta^{2}$ was considered small if $\eta^{2}<0.04$, and large if $\eta^{2}>0.36$. Parametric datasets are summarized in text as mean (SD) or (SEM). All statistical analysis was performed using Statistical Analysis Statistical analysis IBM SPSS Statistics version 24.0 (Chicago, IL, USA).

\section{RESULTS}

Recruitment began September 1, 2016, and closed June 30, 2017. The final follow-up visit was in July 2017. Of 70 participants who entered the run-in phase, 56 (80\%) were randomized. Reasons for pre-randomization withdrawal included BMI to high, refuse to participate, or a medical condition (Figure 1). Five participants (two from the control group, two from the RT group, and one from the combined group) were excluded from blood samples analyses because serum was technically inadequate for hormonal analysis.

Table 2 shown baseline participant characteristics. No significant intergroup baseline differences were observed. 


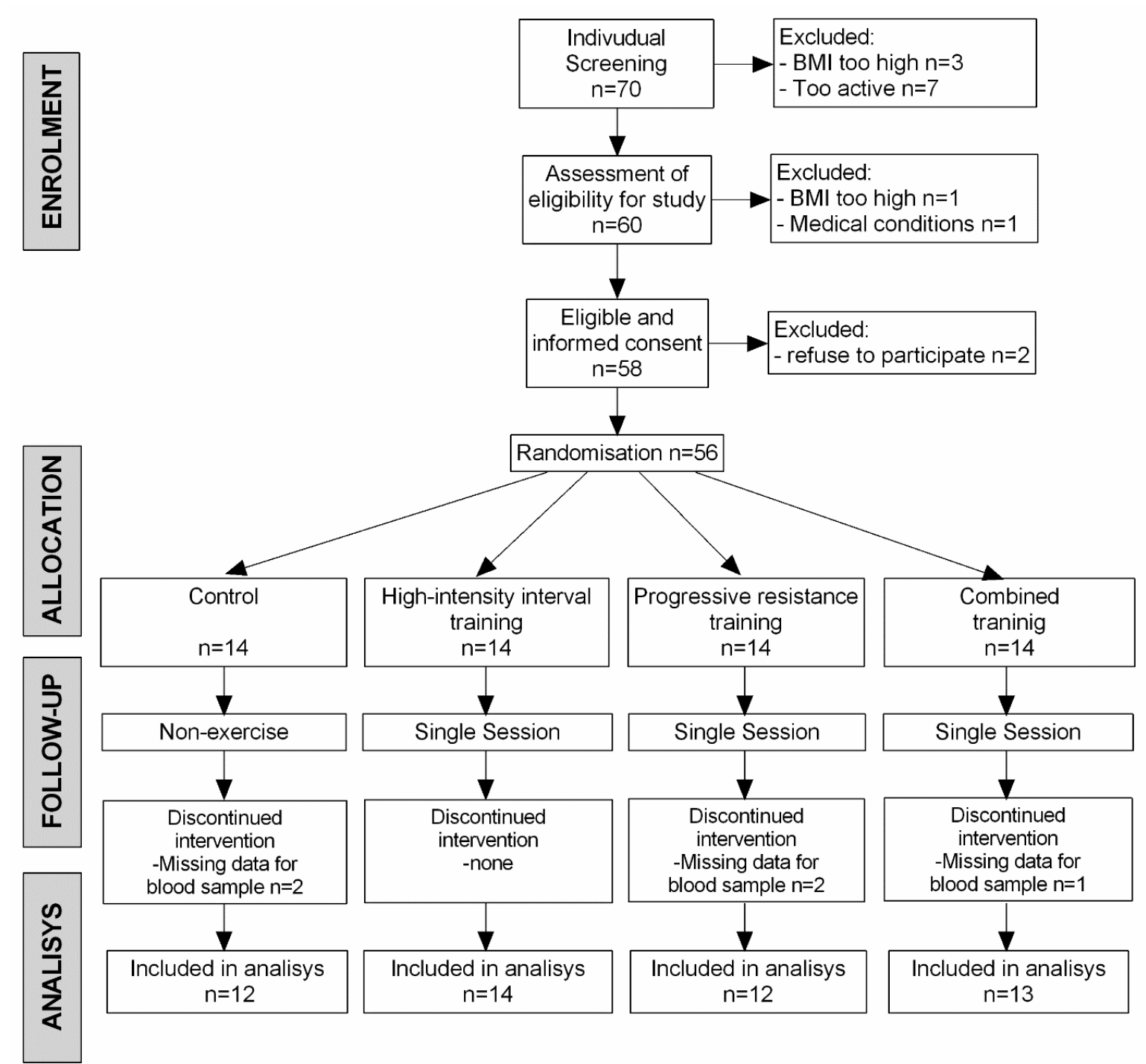

Figure 1. The BrainFit Trial flow diagram. Figure legend. Those whose blood samples $(n=5)$ were technically inadequate and not analyzed. BMI, body mass index.

Acute hormonal response results are displayed in Figure 2 to Figure 5. Decreases in cortisol levels were $-57.08\left(95 \% \mathrm{CI},-75,58\right.$ to $\left.-38,58 ; \mathrm{P}=0.001 ; \eta^{2}=0.61\right)$ and $-37.65(95 \% \mathrm{CI}$, $-54,36$ to $-20,93 ; \mathrm{P}=0.001 ; \eta^{2}=0.51$ ) in the HIIT and control group, respectively (Figure 2). Increases in $\mathrm{T} / \mathrm{C}$ ratio were $0.022\left(95 \% \mathrm{CI}, 0.012\right.$ to $\left.0.031 ; \mathrm{P}=0.001 ; \eta^{2}=0.49\right)$ and 0.015 (95\%CI, 0.004 to $\left.0.025 ; \mathrm{P}=0.007 ; \eta^{2}=0.29\right)$ in the HIIT and control group, respectively (Figure 5). 
Table 2. Baseline participant characteristics by group treatment.

\begin{tabular}{lcccc}
\hline & \multicolumn{4}{c}{ Groups } \\
\cline { 2 - 5 } Characteristics & $\begin{array}{c}\text { Control } \\
(\mathrm{n}=14)\end{array}$ & $\begin{array}{c}\text { HIIT } \\
(\mathrm{n}=14)\end{array}$ & $\begin{array}{c}\text { RT } \\
(\mathrm{n}=14)\end{array}$ & $\begin{array}{c}\text { HIIT+RT } \\
(\mathrm{n}=14)\end{array}$ \\
\hline Age, $\mathrm{yr}$ & $24.75(3.44)$ & $24.50(3.76)$ & $22.85(3.16)$ & $22.25(3.41)$ \\
Weight, $\mathrm{kg}$ & $88.64(8.90)$ & $81.75(6.78)$ & $83.98(7.48)$ & $80.65(6.76)$ \\
Height, m & $1.75(0.05)$ & $1.72(0.05)$ & $1.68(0.18)$ & $1.69(0.05)$ \\
BMI, kg/m ${ }^{2}$ & $28.75(2.01)$ & $27.43(1.74)$ & $27.86(1.31)$ & $28.18(1.25)$ \\
Waist circumference, cm & $97.96(6.31)$ & $95.36(4.91)$ & $94.18(4.68)$ & $96.93(5.80)$ \\
Body fat percentage, $\%$ & $28.75(4.16)$ & $26.21(4.39)$ & $27.08(3.78)$ & $28.15(3.65)$ \\
VO ${ }_{2}$ max, ml·kg. min ${ }^{-1}$ & $41.22(17.36)$ & $40.61(16.76)$ & $38.97(10.55)$ & $37.88(13.62)$ \\
EE during training, kcal & N.A & $462.69(74.91)$ & $460.91(86.77)$ & $461.70(59.12)$ \\
Total testosterone, $\mathrm{ng} / \mathrm{ml}$ & $4.30(1.71)$ & $4.44(1.00)$ & $4.75(1.96)$ & $4.73(0.96)$ \\
Free testosterone, pg/ml & $12.97(6.96)$ & $13.67(3.07)$ & $16.23(5.20)$ & $16.04(4.56)$ \\
Cortisol, ng/ml & $120.48(44.73)$ & $151.14(27.86)$ & $147.11(25.97)$ & $147.54(37.39)$ \\
T/C ratio & $0.042(0.027)$ & $0.031(0.009)$ & $0.034(0.015)$ & $0.034(0.010)$ \\
\hline
\end{tabular}

Mean (standard deviation). HIIT, high-intensity interval training; RT, resistant training; BMI, body mass index; EE, energy expenditure; $\dot{\mathrm{VO}}_{2} \mathrm{max}$, cardiorespiratory fitness; T/C, total-testosterone/cortisol-ratio; NA, not applicable

In per-protocol analyses, differences between groups over time were noted for: Cortisol (control vs RT, $\mathrm{P}=0.002$ ), (control vs HIIT+RT, $\mathrm{P}=0.001$ ), (HIIT vs HIIT+RT, $\mathrm{P}=0.001$ ), (HIIT vs RT, $\mathrm{P}=0.002$ ), interaction effect $\mathrm{F}$ (7.777), $\eta^{2}=0.33$; and $\mathrm{T} / \mathrm{C}$ ratio (control vs $\mathrm{RT}$, $\mathrm{P}=0.011$ ), (control vs HIIT+RT, $\mathrm{P}=0.019$ ), (HIIT vs HIIT+RT, $\mathrm{P}=0.006$ ), (HIIT vs RT, $\mathrm{P}=0.003)$, interaction effect $\mathrm{F}(5.298), \mathrm{\eta}^{2}=0.25$. 

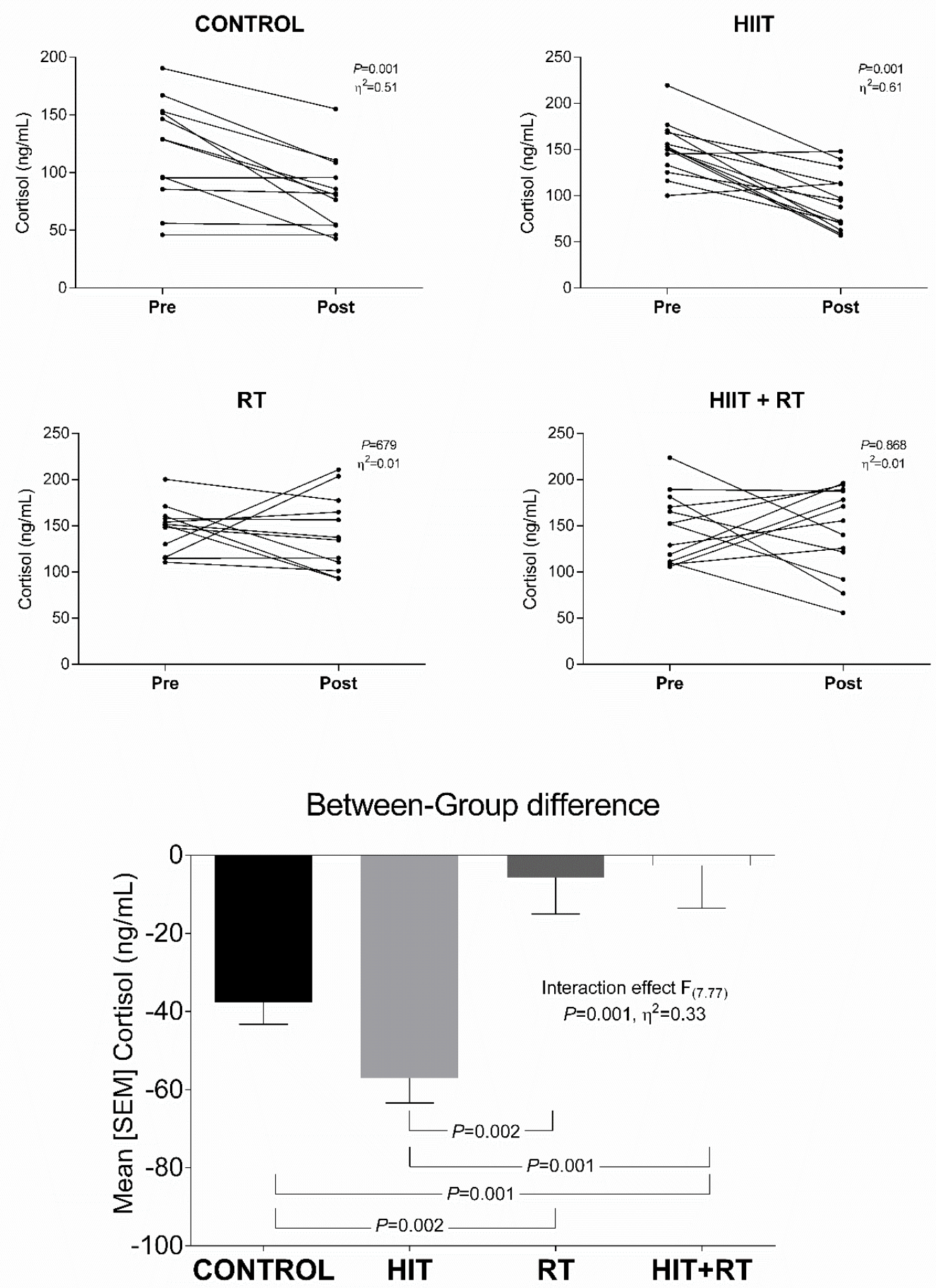

Figure 2. Acute effects by group treatment on cortisol responses in inactive overweight individuals 
CONTROL

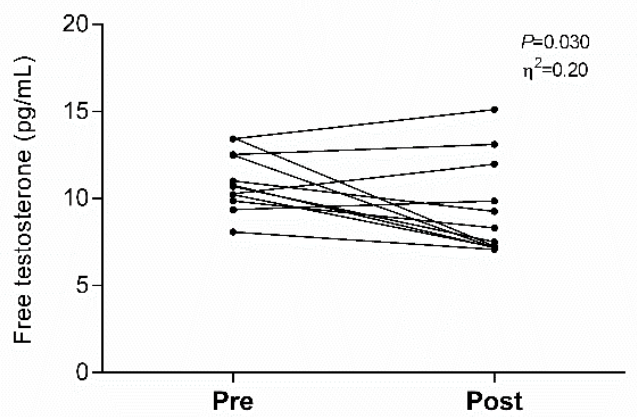

RT

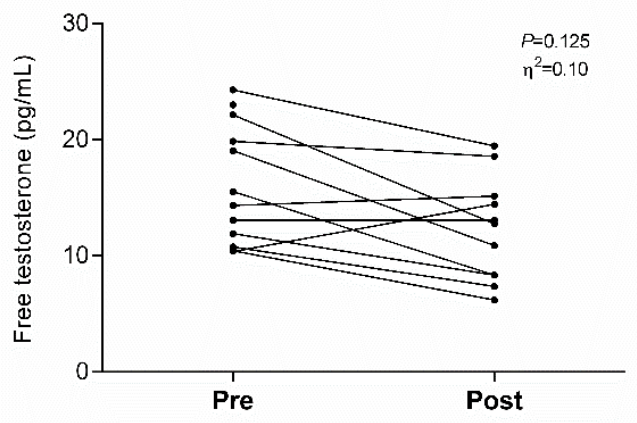

HIIT

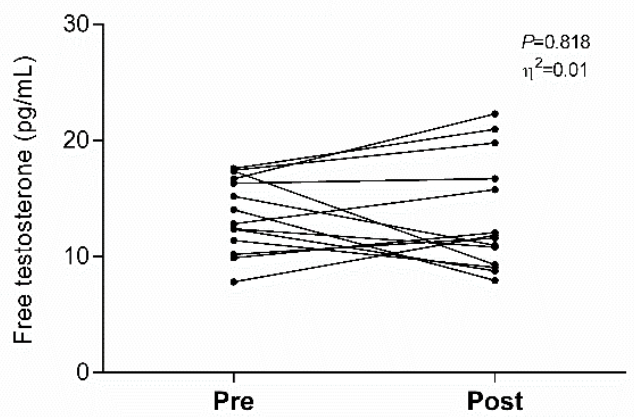

HIIT + RT

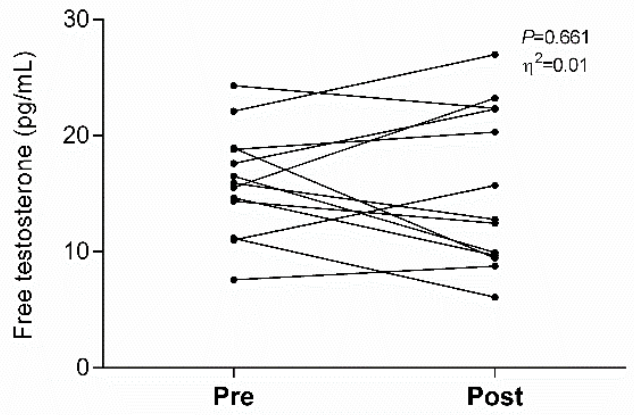

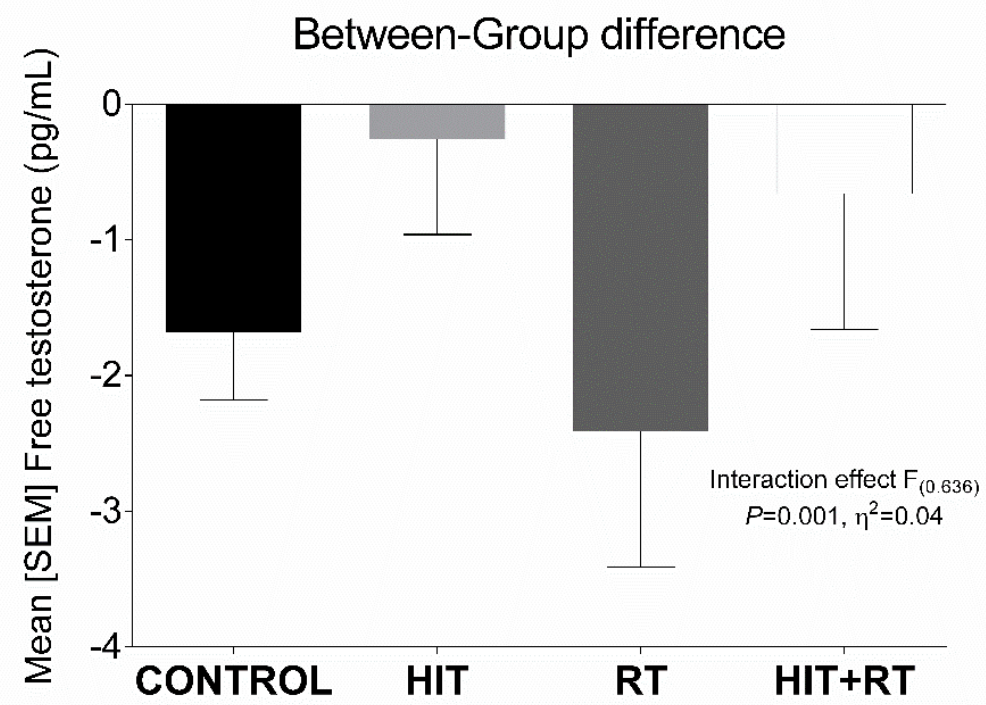

Figure 3. Acute effects by group treatment on free testosterone responses in inactive overweight individuals 
CONTROL

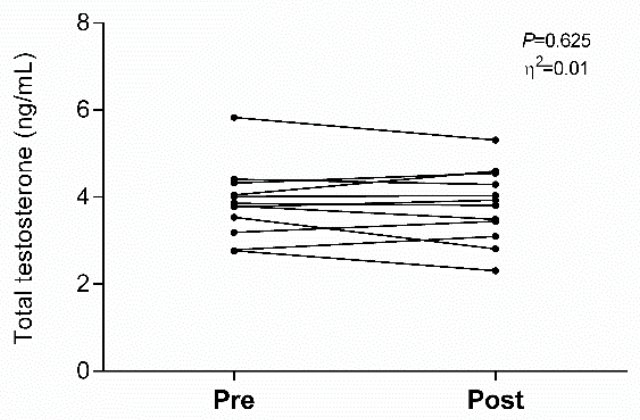

RT

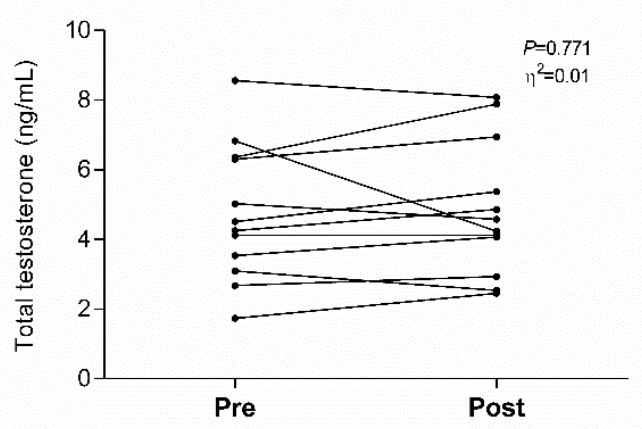

HIIT

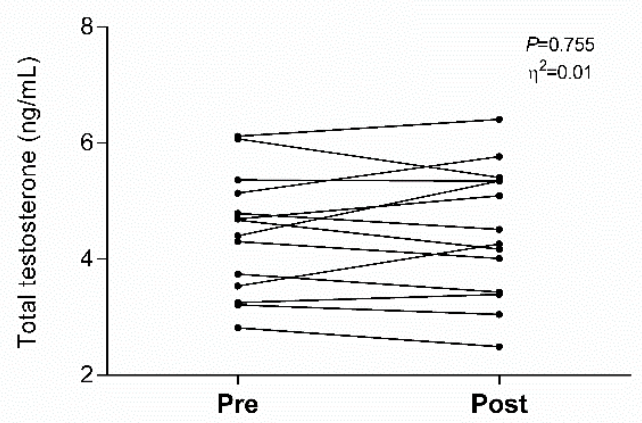

HIIT + RT

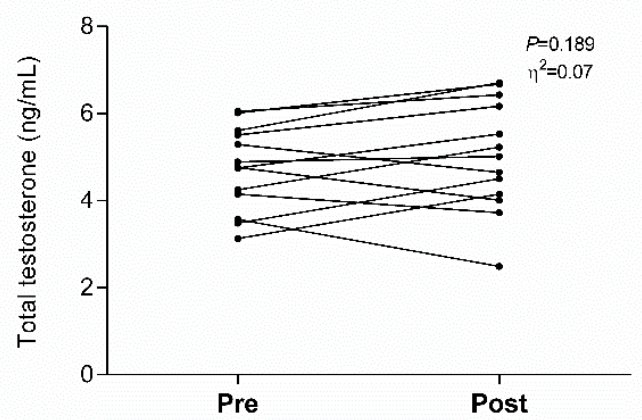

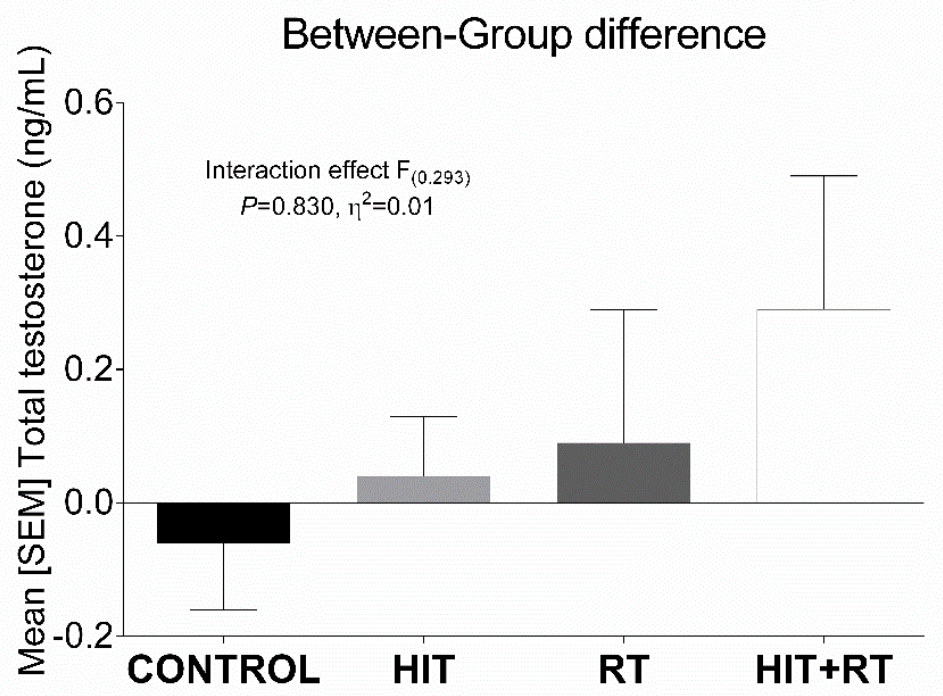

Figure 4. Acute effects by group treatment on total testosterone responses in inactive overweight individuals 
CONTROL

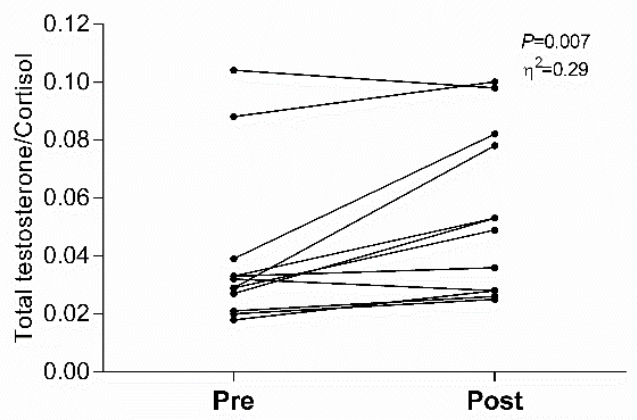

RT

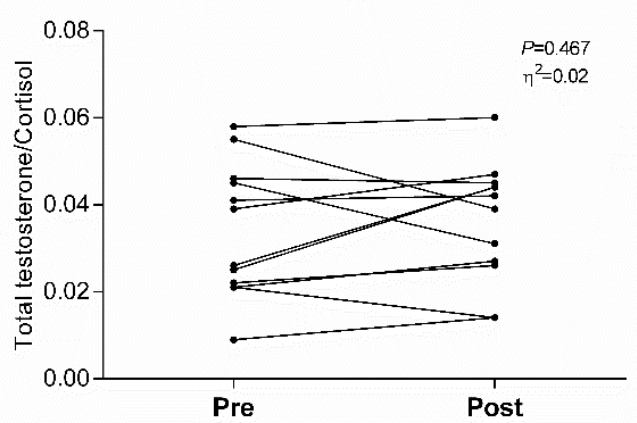

HIIT

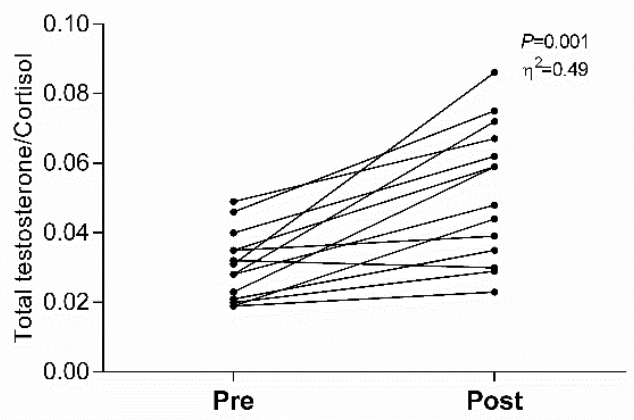

HIIT + RT

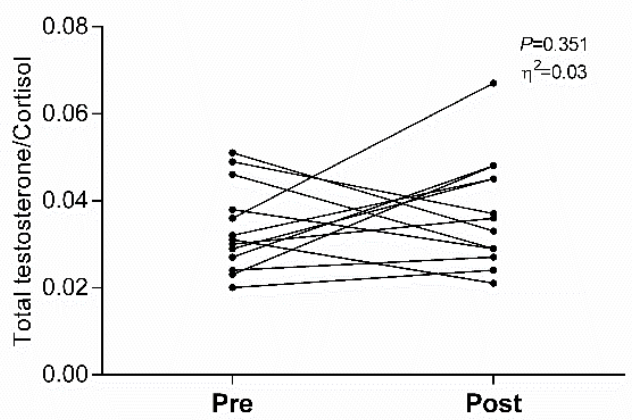

\section{Between-Group difference}

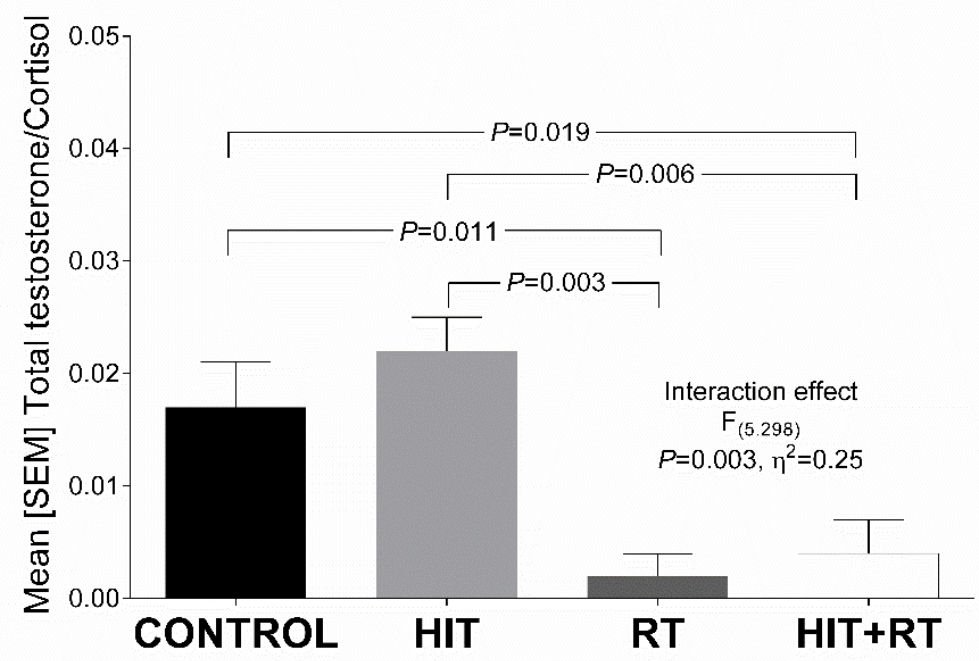

Figure 5. Acute effects by group treatment on total testosterone/cortisol ratio responses in inactive overweight individuals 


\section{DISCUSION}

The main findings of the present study were the HIIT protocol induced marked decreases in the serum cortisol concentrations in overweight young male adults, which was not observed following RT and combined protocols. In addition, HIIT protocol induced significant increase in the $\mathrm{T} / \mathrm{C}$ ratio in these individuals, which also did not occur following the other protocols.

Previous studies have shown a correlation between the acute response of testosterone to single training sessions and the magnitude of the increase in strength, power and muscle mass resulting from chronic strength training adaptations $(26,27)$. Besides the important role of testosterone in the synthesis of contractile proteins $(28,29)$, as well as in the synthesis of neurotransmitters related to strength production (26), the increase in testosterone in response to strength training seems to be related to the magnitude of the increase in the number of androgen receptors (AR) in skeletal muscle human cells (28). Additionally, it has been proposed that sex hormone globulin binding (SHBG) or androgen receptors increase after resistance exercise in obese male (5).

Other studies in animal models demonstrated that the amount of ARs plays an important role in the magnitude of muscle adaptations to training (29). Thus, the manipulation of the various factors related to the training session that influence acute hormonal responses, such as volume and intensity, time interval and muscle mass involved can optimize the increase in testosterone in response to the training session and up-regulate AR expression; thereby, amplifying the anabolic effects (26).

Regarding aerobic training, although the importance of the anabolic hormonal response is not well known; testosterone and cortisol seem to be more responsive to higher intensity exercise $(30,31)$. Therefore, it is not clear why the HIIT protocol performed in the present study did not elicit a significant elevation in testosterone level. This could be explained by the measurement time; previous studies have been reported peak testosterone concentration between 0 and 10 minutes after exercise $(11,28)$. Hormonal response to aerobic exercise can be explained in part by increased sympathetic activity during exercise (32) or even by 
greater vasodilation in the testes stimulated by increased release of nitric oxide resulting from the exercise (33). Thus, because obesity is also related with damage in endothelial function, it is possible that the mechanism related to vasodilatation in testes may be at least partially impaired and possibly altering the overall endocrine response (7).

On the other hand, some authors have shown that anaerobic exercise (i.e., strength training, anaerobic running or cycling) seems to be a powerful stimulus to increases in testosterone and cortisol levels (25), which can be explained by the marked influence of the anaerobic glycolytic pathway in stimulating acute hormonal increases in response to exercise (34). Therefore, it could be expected that both HIIT, and RT sessions used in the present study, in which the glycolytic pathway is strongly activated, have a great potential for stimulating testosterone.

In a study by Lu et al. (34), a correlation between the increase in testosterone and increase in lactate during an incremental protocol was observed in rats. Furthermore, these authors demonstrated in vitro that the direct infusion of lactate in the testes resulted in a dosedependent increase in testosterone. The androgen receptor (AR) content was not measured in the present study, and it is possible that the non-significant increase in the circulating total testosterone and decrease in the free testosterone might have had influence of the increased hormone-receptor interaction. Furthermore, it is well known that the concentrational peak of testosterone occurs around 8 am and decreases to its lowest levels at about 4 pm by circadian cycle regulation (35). Therefore, the non-significant changes of serum testosterone might have been a result of increased receptor binding and clearance, and not simply a lack of increased production. Interestingly, Willoughby and Taylor (28) found positive correlation between acute response of testosterone and increase number of androgenic receptors after resistance protocol $(r=0.89, p=<0.05)$.

However, this hypothesis should be considered with caution and remains speculative. Another explanation to the non-significant changes on testosterone levels is due to overweight/obesity states inducing hormonal abnormalities (3), and specifically testosterone and cortisol are greatly affected by obesity (36). Future studies comparing 
testosterone, androgenic receptors, SHBG responses to exercise measured during the first hour post-exercise between healthy-weight and overweight or obese individuals performing the same protocol are needed to determine whether in fact adiposity may impair increases in testosterone in response to exercise. Finally, while the intensity of the exercise is critical to evoking a hormonal response, so is the dosage of the exercise (i.e. average intensity $\mathrm{x}$ total duration), thus perhaps the duration component in our exercise was insufficient. Wilkerson et al. used a short-term continuous exercise protocol, not HIIT, but alluded to this possibility in their work on exercise testosterone responses (37).

With respect to circulating cortisol, a decrease was observed after the HIIT protocol, which disagrees with studies investigating the responses of this hormone after high-intensity exercise bouts. Specifically, investigators observed no changes after moderate interval training of rowing (38), or even acute increases after HIIT at cycle ergometer (39). Cortisol, which is responsible for $95 \%$ of glucocorticoid activity, is a catabolic hormone responsible for lipid and protein degradation and subsequent mobilization of energy substrate during exercise (40). Although several studies investigated the cortisol acute responses to aerobic and strength training bouts in healthy-weight individuals $(3,30,36,41)$, there is less information regarding the cortisol responses to HIIT, RT and combined training in overweight male individuals.

Because the role of cortisol in mobilizing energy subtract during exercise, it is not clear why our subjects significantly reduced their cortisol levels following HIIT training, and the same was not observed when observing RT and combined training, even equalizing the total energy expenditure between the exercise sessions. In our study, a decrease in cortisol levels was observed in the control group, which could be attributed at circadian cycle, taking into account the peak concentration occurs around 8 am and its progressive decline until reaching the lowest values around $8 \mathrm{pm}$. (35).

Based on the present results regarding the reduction of cortisol levels after HIIT training (as we implemented), in order to induce a less catabolic response to exercise, HIIT may be suggested as an alternative to improve cardiorespiratory fitness without excessive increases 
the catabolic state, since it has been shown that continuous aerobic training promotes greater increases in cortisol levels (37). Indeed, some studies have shown that the use of pharmacological cortisol has an inhibitory effect on the steroidogenic process in Leydig cells, via enzymatic inhibition (42) or by eliminating cAMP production (39).

Our findings may suggest that the acute response of cortisol may be favorable in overweight and obese individuals following HIIT or, otherwise suggest that obese individuals have an impaired hypothalamic-pituitary-adrenal axis. The purpose of this study was to investigate whether there are differences in the acute testosterone, cortisol and T/C ratio following HIIT, RT and combined training bouts with similar energy expenditure in overweight individuals. Indeed, our results have shown that HIIT protocol induced a significant increase in the T/C ratio, which appear to be entirely due to the decrease observed in cortisol levels and the maintenance of testosterone levels. One could suggest that this positive change in the T/C ratio is favorable to muscle adaptations induced by HIIT because of more anabolic environment, but the role of this hormonal ratio as an indicator of muscle adaptations induced by a training is not entirely understood and should be further investigated.

One possible limitation of this study is the absence of continuous aerobic training and combined strength and continuous aerobic training bouts to compare to the exercise protocols investigated. In addition, we investigated the testosterone, cortisol and T/C ratio using specific volume and intensity and our results should not be extrapolated to others exercise protocols.

Moreover, in what extent the acute hormone responses observed in the present study could explain chronic adaptations to training needs to be investigated in a long-term study. On the other hand, this appears to be the first study to compare the acute testosterone and cortisol responses to HIIT, RT and combined HIIT and RT exercise bouts with similar energy expenditure in overweight individuals with a relatively robust sample size. 
In conclusion, the results of this study showed that the HIIT protocol induced a decrease in the cortisol levels as well as an increase in the T/C ratio, whereas non-significant changes were observed following RT or combined HIIT and RT bout. Based on the relationship, suggested in the literature, between acute hormonal responses and chronic neuromuscular adaptations to strength training, this could be positive for the optimization of long term muscle adaptations. However, the extent of the importance of this response in chronic adaptations to HIIT remains speculative and further studies trying to elucidate hormonal acute responses to HIIT adaptations in overweight individuals are needed to determine whether the meaning of these acute responses.

\section{REFERENCES}

1. Herrán OF, Patiño GA, Del Castillo SE. Dietary transition and excess weight in adults according to the Encuesta de la Situación Nutricional en Colombia, 2010. Biomedica. 2016;36(1):109-20.

2. Kohl HW, Craig CL, Lambert EV, Inoue S, Alkandari JR, Leetongin G, et al. The pandemic of physical inactivity: global action for public health. Lancet. 2012;380(9838):294-305.

3. Bekaert M, Van Nieuwenhove Y, Calders P, Cuvelier CA, Batens AH, Kaufman $\mathrm{JM}$, et al. Determinants of testosterone levels in human male obesity. Endocrine. 2015;50(1):202-11.

4. Vandewalle S, De Schepper J, Kaufman JM. Androgens and obesity in male adolescents. Curr Opin Endocrinol Diabetes Obes. 2015;22(3):230-7.

5. O'Leary CB, Hackney AC. Acute and chronic effects of resistance exercise on the testosterone and cortisol responses in obese males: a systematic review. Physiol Res. 2014;63(6):693-704.

6. Akishita M, Hashimoto M, Ohike Y, Ogawa S, Iijima K, Eto M, et al. Low testosterone level as a predictor of cardiovascular events in Japanese men with coronary risk factors. Atherosclerosis. 2010;210(1):232-6.

7. Rubin DA, Hackney AC. Inflammatory cytokines and metabolic risk factors during growth and maturation: influence of physical activity. Med Sport Sci. 2010;55:43-55.

8. Buch A, Kis O, Carmeli E, Keinan-Boker L, Berner Y, Barer Y, et al. Circuit resistance training is an effective means to enhance muscle strength in older and middle aged adults: A systematic review and meta-analysis. Ageing Res Rev. 2017;37:16-27.

9. Sylta $\varnothing$, Tønnessen E, Sandbakk Ø, Hammarström D, Danielsen J, Skovereng K, et al. Effects of High-Intensity Training on Physiological and Hormonal Adaptions in WellTrained Cyclists. Med Sci Sports Exerc. 2017;49(6):1137-46.

10. Mangine GT, Hoffman JR, Gonzalez AM, Townsend JR, Wells AJ, Jajtner AR, et al. Exercise-Induced Hormone Elevations Are Related to Muscle Growth. J Strength Cond Res. 2017;31(1):45-53. 
11. Zinner C, Wahl P, Achtzehn S, Reed JL, Mester J. Acute hormonal responses before and after 2 weeks of HIT in well trained junior triathletes. Int $\mathrm{J}$ Sports Med. 2014;35(4):316-22.

12. Wahl P, Mathes S, Köhler K, Achtzehn S, Bloch W, Mester J. Acute metabolic, hormonal, and psychological responses to different endurance training protocols. Horm Metab Res. 2013;45(11):827-33.

13. Viru A, Viru M. Cortisol--essential adaptation hormone in exercise. Int J Sports Med. 2004;25(6):461-4.

14. West DW, Burd NA, Tang JE, Moore DR, Staples AW, Holwerda AM, et al. Elevations in ostensibly anabolic hormones with resistance exercise enhance neither training-induced muscle hypertrophy nor strength of the elbow flexors. J Appl Physiol (1985). 2010;108(1):60-7.

15. Hoogeveen AR, Zonderland ML. Relationships between testosterone, cortisol and performance in professional cyclists. Int J Sports Med. 1996;17(6):423-8.

16. Chan J, Sauvé B, Tokmakejian S, Koren G, Van Uum S. Measurement of cortisol and testosterone in hair of obese and non-obese human subjects. Exp Clin Endocrinol Diabetes. 2014;122(6):356-62.

17. Sheikholeslami-Vatani D, Siahkouhian M, Hakimi M, Ali-Mohammadi M. The effect of concurrent training order on hormonal responses and body composition in obese men. Science \& Sports. 2015;30(6):335-41.

18. McMurray RG, Hackney AC. Interactions of metabolic hormones, adipose tissue and exercise. Sports Med. 2005;35(5):393-412.

19. (WHO) WHO. Global Strategy on Diet, Physical Activity and Health [Available from: http://www.who.int/dietphysicalactivity/factsheet_adults/en/.

20. Services UDoHaH. Physical Activity Guidelines AdvisoryCommittee. Physical Activity Guidelines Advisory Committee Report. [Available from: http://www.health.gov/paguidelines/.

21. Ramírez-Vélez R, Hernandez A, Castro K, Tordecilla-Sanders A, González-Ruíz K, Correa-Bautista JE, et al. High Intensity Interval- vs Resistance or Combined- Training for Improving Cardiometabolic Health in Overweight Adults (Cardiometabolic HIIT-RT Study): study protocol for a randomised controlled trial. Trials. 2016;17(1):298.

22. Graf S, Karsegard VL, Viatte V, Maisonneuve N, Pichard C, Genton L. Comparison of three indirect calorimetry devices and three methods of gas collection: a prospective observational study. Clin Nutr. 2013;32(6):1067-72.

23. Organization WH. Obesity: preventing and managing the global epidemic. Reportof a WHO consultation on obesity. 1997.

24. Marfell-Jones M OT, Stewart, A. International Standards for AnthropometricAssessment; ISAK: Potchefstroom, South Africa. 2006.

25. Rodríguez-Rodríguez F, Cristi-Montero C, González-Ruíz K, Correa-Bautista JE, Ramírez-Vélez R. Bioelectrical Impedance Vector Analysis and Muscular Fitness in Healthy Men. Nutrients. 2016;8(7).

26. Kraemer WJ, Ratamess NA. Hormonal responses and adaptations to resistance exercise and training. Sports Med. 2005;35(4):339-61.

27. Cadore EL, Lhullier FL, Brentano MA, da Silva EM, Ambrosini MB, Spinelli R, et al. Hormonal responses to resistance exercise in long-term trained and untrained middleaged men. J Strength Cond Res. 2008;22(5):1617-24. 
28. Willoughby DS, Taylor L. Effects of sequential bouts of resistance exercise on androgen receptor expression. Med Sci Sports Exerc. 2004;36(9):1499-506.

29. Inoue K, Yamasaki S, Fushiki T, Kano T, Moritani T, Itoh K, et al. Rapid increase in the number of androgen receptors following electrical stimulation of the rat muscle. Eur J Appl Physiol Occup Physiol. 1993;66(2):134-40.

30. Galbo H, Hummer L, Peterson IB, Christensen NJ, Bie N. Thyroid and testicular hormone responses to graded and prolonged exercise in man. Eur J Appl Physiol Occup Physiol. 1977;36(2):101-6.

31. Hackney AC. Testosterone, the hypothalamo-pituitary-testicular axis, and endurance exercise training: A review. Biology of Sport , ; 1996. p. 85 - 98.

32. Fahrner C, Hackney A. Effects of endurance exercise on free testosterone concentration and the binding affinity of sex hormone binding globulin (SHBG). International journal of sports medicine. 1998;19(01):12-5.

33. Meskaitis V, Harman F, Volek J, Nindl B, Kraemer W, Weinstok D, et al. Effects of exercise on testosterone and nitric oxide production in the rats testis. J Androl Suppl. $1997 ; 31$.

34. Lu SS, Lau CP, Tung YF, Huang SW, Chen YH, Shih HC, et al. Lactate and the effects of exercise on testosterone secretion: evidence for the involvement of a cAMPmediated mechanism. Med Sci Sports Exerc. 1997;29(8):1048-54.

35. van Kerkhof LW, Van Dycke KC, Jansen EH, Beekhof PK, van Oostrom CT, Ruskovska T, et al. Diurnal Variation of Hormonal and Lipid Biomarkers in a Molecular Epidemiology-Like Setting. PLoS One. 2015;10(8):e0135652.

36. Sato K, Iemitsu M. Exercise and sex steroid hormones in skeletal muscle. J Steroid Biochem Mol Biol. 2015;145:200-5.

37. Wilkerson JE, Horvath SM, Gutin B. Plasma testosterone during treadmill exercise. J Appl Physiol Respir Environ Exerc Physiol. 1980;49(2):249-53.

38. Kokalas N, Tsalis G, Tsigilis N, Mougios V. Hormonal responses to three training protocols in rowing. Eur J Appl Physiol. 2004;92(1-2):128-32.

39. Hough JP, Papacosta E, Wraith E, Gleeson M. Plasma and salivary steroid hormone responses of men to high-intensity cycling and resistance exercise. J Strength Cond Res. 2011;25(1):23-31.

40. Welsh TH, Bambino TH, Hsueh AJ. Mechanism of glucocorticoid-induced suppression of testicular androgen biosynthesis in vitro. Biol Reprod. 1982;27(5):1138-46.

41. Cadore EL, Izquierdo M, dos Santos MG, Martins JB, Rodrigues Lhullier FL, Pinto RS, et al. Hormonal responses to concurrent strength and endurance training with different exercise orders. J Strength Cond Res. 2012;26(12):3281-8.

42. Cumming DC, Quigley ME, Yen SS. Acute suppression of circulating testosterone levels by cortisol in men. J Clin Endocrinol Metab. 1983;57(3):671-3. 